\title{
Nonlocal electrodynamics of linearly accelerated systems
}

\author{
Bahram Mashhoon \\ Department of Physics and Astronomy, University of Missouri-Columbia, Columbia, Missouri 65211, USA
}

(Received 30 July 2004; published 8 December 2004)

\begin{abstract}
The measurement of an electromagnetic radiation field by a linearly accelerated observer is discussed. The nonlocality of this process is emphasized. The nonlocal theory of accelerated observers is briefly described and the consequences of this theory are illustrated using a concrete example involving the measurement of an incident pulse of radiation by an observer that experiences uniform acceleration during a limited interval of time.
\end{abstract}

DOI: 10.1103/PhysRevA.70.062103

PACS number(s): 03.30.+p, 11.10.Lm, 04.20.Cv

\section{INTRODUCTION}

Imagine an inertial frame in Minkowski spacetime in which the fundamental static inertial observers measure the electric field $\mathbf{E}(t, \mathbf{x})$ and magnetic field $\mathbf{B}(t, \mathbf{x})$. The fields measured by an inertial observer moving with uniform velocity $\mathbf{v}$ are then given by (see, e.g., [1])

$$
\begin{gathered}
\mathbf{E}_{\|}^{\prime}=\mathbf{E}_{\|}, \quad \mathbf{B}_{\|}^{\prime}=\mathbf{B}_{\|}, \\
\mathbf{E}_{\perp}^{\prime}=\gamma\left(\mathbf{E}+\frac{\mathbf{v}}{c} \times \mathbf{B}\right)_{\perp}, \quad \mathbf{B}_{\perp}^{\prime}=\gamma\left(\mathbf{B}-\frac{\mathbf{v}}{c} \times \mathbf{E}\right)_{\perp},
\end{gathered}
$$

where $\gamma$ is the Lorentz factor of the observer. Equations (1) and (2) are obtained from the fact that Maxwell's equations are invariant under Lorentz transformations. More explicitly, let us define the Faraday tensor $F_{\mu \nu} \rightarrow(\mathbf{E}, \mathbf{B})$ such that $F_{0 i}$ $=-E_{i}$ and $F_{i j}=\epsilon_{i j k} B^{k}$. Greek indices run from 0 to 3 and latin indices run from 1 to 3 . Under a Poincaré transformation $x^{\prime \mu}=L_{\nu}^{\mu} x_{\nu}{ }^{\nu} \alpha^{\mu}$, where $L^{\mu}{ }_{\nu}$ is a Lorentz matrix and $\alpha^{\mu}$ represents a spacetime translation,

$$
F^{\prime \mu \nu}\left(\mathbf{x}^{\prime}\right)=\frac{\partial x^{\prime \mu}}{\partial x^{\rho}} \frac{\partial x^{\prime \nu}}{\partial x^{\sigma}} F^{\rho \sigma}(\mathbf{x}) .
$$

In component form, this expression reduces to Eqs. (1) and (2).

The basic laws of microphysics have been formulated with respect to ideal inertial observers. However, all actual observers are accelerated. It is therefore necessary to determine what accelerated observers measure. The term "observer" is employed in this paper in an extended sense; for instance, an observer could be an ideal measuring device. In the theory of measurement, it is important to distinguish the practical aspects of a measurement from its basic theoretical aspects. Clearly a measuring device has limitations due to the nature of its construction as well as its modes of operation. These limitations need to be taken into account when using such a device in the laboratory. On the other hand, what is at issue here is our theoretical expectation of an ideal device of this kind when it is accelerated. To make contact with the basic laws of microphysics, the accelerated device must be related via a theoretical postulate to ideal inertial devices. The postulate that is employed in the standard theory of relativity is the hypothesis of locality [2-6], namely, the assumption that the accelerated observer is lo- cally equivalent to an otherwise identical momentarily comoving inertial observer. Along the worldline, the accelerated observer passes through a continuous infinity of such hypothetical momentarily comoving inertial observers. To implement this hypothesis of locality, one must therefore consider a class of Lorentz transformations between the global background inertial frame and the inertial frames of the momentarily comoving inertial observers. It is convenient, however, to adopt a different, but physically equivalent, approach based on the use of orthonormal tetrads.

Each inertial observer is endowed with an orthonormal tetrad $\lambda_{(\alpha)}^{\mu}$, where $\lambda_{(0)}^{\mu}$ is the temporal axis of the observer and $\lambda_{(i)}^{\mu}, i=1,2,3$, are the spatial axes of the observer. The measurement of the electromagnetic field by the inertial observer amounts to the projection of the Faraday tensor on the observer's tetrad

$$
F_{(\alpha)(\beta)}=F_{\mu \nu} \lambda_{(\alpha)}^{\mu} \lambda_{(\beta)}^{\nu} .
$$

For the fundamental set of static inertial observers in the background global inertial frame, $\lambda_{(\alpha)}^{\mu}=\delta^{\mu}{ }_{\alpha}$ and hence from Eq. (4), $\mathbf{E}$ and $\mathbf{B}$ have the interpretation of fields measured by these observers. For an inertial observer moving with velocity v, Eq. (4) reduces to Eqs. (1) and (2), as expected. To see this explicitly in the case of motion along the $z$ axis, we note that the tetrad of such an inertial observer is

$$
\begin{aligned}
& \lambda_{(0)}^{\mu}=\gamma(1,0,0, \beta), \quad \lambda_{(1)}^{\mu}=(0,1,0,0), \\
& \lambda_{(2)}^{\mu}=(0,0,1,0), \quad \lambda_{(3)}^{\mu}=\gamma(\beta, 0,0,1),
\end{aligned}
$$

where $\beta=v / c$ and $\gamma=\left(1-\beta^{2}\right)^{-1 / 2}$ is the Lorentz factor. Equation (4) then implies that

$$
\begin{array}{cl}
E_{1}^{\prime}=\gamma\left(E_{1}-\beta B_{2}\right), & B_{1}^{\prime}=\gamma\left(B_{1}+\beta E_{2}\right), \\
E_{2}^{\prime}=\gamma\left(E_{2}+\beta B_{1}\right), & B_{2}^{\prime}=\gamma\left(B_{2}-\beta E_{1}\right), \\
E_{3}^{\prime}=E_{3}, & B_{3}^{\prime}=B_{3},
\end{array}
$$

in agreement with Eqs. (1) and (2).

It follows from the hypothesis of locality that an accelerated observer is also endowed with an orthonormal tetrad $\lambda_{(\alpha)}^{\mu}(\tau)$, where $\tau$ is the proper time along its worldline $x^{\mu}$ $=x^{\mu}(\tau)$, 


$$
\tau=\int_{0}^{t} \sqrt{1-\beta^{2}} d t
$$

Here it has been assumed that $\tau=0$ at $t=0$. The hypothesis of locality implies that $\tau$ is the time recorded by an ideal clock comoving with the accelerated observer. The question then arises whether the field measured by the accelerated observer is point by point given by Eq. (4) as required by the hypothesis of locality. To answer this question, we must first study the limitations of the hypothesis of locality.

Consider, for instance, the measurement of the frequency of a plane monochromatic electromagnetic wave of frequency $\omega$ and wave vector $\mathbf{k}$ by an observer moving with a velocity $\mathbf{v}(t)$. Assuming that at each instant of time $t$, the accelerated observer is equivalent to an inertial observer with the same instantaneous velocity, the invariance of the phase of the wave under Lorentz transformations implies that the frequency $\omega^{\prime}$ measured by the observer is given by the Doppler effect

$$
\omega^{\prime}=\frac{\omega-\mathbf{k} \cdot \mathbf{v}(t)}{\sqrt{1-v^{2} / c^{2}}} .
$$

This frequency depends on the state of motion of the observer; therefore, Eq. (10) makes physical sense if the observer is able to measure the frequency $\omega^{\prime}$ during a period of time in which $\mathbf{v}(t)$ does not change appreciably. Since the observer needs to record at least a few periods of the wave in order to measure its frequency, Eq. (10) is physically reasonable if

$$
n \frac{2 \pi}{\omega}\left|\frac{d \mathbf{v}(t)}{d t}\right| \ll|\mathbf{v}(t)|,
$$

where $n \sim 1$. Here $n$ is the number of cycles of the wave used to measure its frequency. In general, the frequency can be determined more accurately if more cycles are employed; for large $n$, however, one finds from Eq. (11) that the acceleration must then be very small. Combining Eq. (11) with $|\mathbf{v}|$ $<c$, we find that $\lambda \ll c^{2} / A$, where $\lambda=\lambda /(2 \pi)$ is the reduced wavelength of the radiation and $A=|d \mathbf{v} / d t|$ is the magnitude of the three-dimensional acceleration vector of the observer. Thus the Doppler formula in general makes sense if the reduced wavelength of the radiation is much smaller than the acceleration length $\mathcal{L}=c^{2} / A$ of the observer. On the other hand, the instantaneous Lorentz transformations may be employed in accordance with the hypothesis of locality to assign electric and magnetic fields to the accelerated observer. These fields may then be Fourier analyzed in the local frame of the observer to determine the frequency spectrum. It is expected that this spectrum would then reduce to the Doppler formula in the JWKB limit.

To summarize, for accelerated systems the standard Doppler formula $\omega(\tau)=-k_{\mu} u^{\mu}(\tau)$, where $k^{\mu}$ is the wave fourvector and $u^{\mu}$ is the four-velocity of the observer, will produce a result that in general varies in time. If the frequency changes from one instant of time to the next, the period of the incident wave must in general be negligibly short in order for the Doppler formula to make physical sense; that is, $\lambda / \mathcal{L} \rightarrow 0$.
As a phenomenological alternative, a time-dependent frequency can also be defined as the inverse proper time between successive peaks [7-10]. This has the advantage of defining the frequency over an extended period of time, the same way it would be measured in practice. Both of these approaches invoke the hypothesis of locality.

These phenomenological approaches can be extended to a gravitational field along the lines indicated in [11]. A discussion of the various limitations on frequency measurements in the Schwarzschild geometry is contained in [12].

The hypothesis of locality originates from Newtonian mechanics, where the state of a particle is given by its position and velocity; the accelerated observer therefore has the same state as the comoving inertial observer, hence they are equivalent. This is analogous to approximating a curve with its tangent line at a point. In fact, if all physical phenomena could be reduced to pointlike coincidences of classical particles and rays of radiation, then the hypothesis of locality would be exactly valid. On the other hand, it is not possible to measure an electric or magnetic field instantaneously, as emphasized by Bohr and Rosenfeld [13,14]. This paper will pursue a nonlocal approach [15-19] that involves in essence an integral averaging of all momentarily equivalent inertial observers for the duration of the acceleration as explained in Sec. II.

The expected deviation from the hypothesis of locality can be expressed in terms of acceleration lengths. In general, the acceleration of the observer is given by an antisymmetric acceleration tensor $\phi_{(\alpha)(\beta)}$ defined by

$$
\frac{d \lambda^{\mu}{ }_{(\alpha)}}{d \tau}=\phi_{(\alpha)}^{(\beta)} \lambda_{(\beta)}^{\mu} .
$$

The translational acceleration $a^{\mu}=d u^{\mu} / d \tau$ is a spacelike vector $\left(u^{\mu} a_{\mu}=0\right)$ and can be expressed as $a^{\mu}=g^{i} \lambda_{(i)}^{\mu}$, where $a^{\mu} a_{\mu}=\mathbf{g} \cdot \mathbf{g}=g^{2}(\tau)$. Here $g(\tau) \geqslant 0$ is the magnitude of the translational acceleration. In analogy with the Faraday tensor, the acceleration tensor may be expressed as $\phi_{(\alpha)(\beta)}$ $\rightarrow(-\mathbf{g}(\tau), \boldsymbol{\Omega}(\tau))$, where $g_{i}(\tau)=\phi_{(0)(i)}$ and $\Omega_{i}(\tau)=\frac{1}{2} \epsilon_{i j k} \phi^{(j)(k)}$. Here $\boldsymbol{\Omega}$ denotes the frequency of rotation of the spatial frame of the accelerated observer with respect to a nonrotating (i.e., Fermi-Walker transported) frame. The invariant acceleration scales are constructed from the scalars $\phi_{(\alpha)(\beta)}$; for instance, the lengths $\mathcal{L}=c^{2} / g$ and $c / \Omega$ and the corresponding acceleration times $c / g$ and $1 / \Omega$ refer to the translational and rotational accelerations of the observer, respectively. The intrinsic acceleration scales determine the scale of variation of the state of the observer; therefore, the hypothesis of locality is a valid approximation if the intrinsic scale of the phenomenon under observation is negligibly small compared to the corresponding acceleration scale of the observer. Thus the deviation from the hypothesis of locality is expected to be proportional to $\lambda / \mathcal{L}$, where $\lambda$ is the intrinsic length scale of the phenomenon under observation. For Earth-based optical experiments in the laboratory, for example, $\lambda \sim 5000 \AA$ for visible light, while $c^{2} / g_{\oplus} \simeq 1 \mathrm{lt}-\mathrm{yr}$ and $c / \Omega_{\oplus} \simeq 28 \mathrm{AU}$; therefore, $\lambda / \mathcal{L}_{\oplus}$ is $\lesssim 10^{-19}$ and any deviations from locality appear to be too small to be detectable at present. On the 
other hand, the development of ultrahigh-power lasers during the past 15 years $[20,21]$ may change the observational situation and could lead to the measurement of deviations from locality.

Nonlocal effects may become detectable with the help of laser pulses that can induce linear electron accelerations of order $10^{24} \mathrm{~cm} / \mathrm{s}^{2}$ using the chirped pulse amplification technique [20,21]. Moreover, Sauerbrey [22] has employed such high-intensity femtosecond lasers to impart linear accelerations of order $10^{21} \mathrm{~cm} / \mathrm{s}^{2}$ to small grains. A grain with a macroscopic mass of $\sim 10^{-12} \mathrm{~g}$ more closely approximates a classical accelerated observer in the sense employed in relativity theory [23].

The nonlocal theory of accelerated observers is discussed in Sec. II. The linearly accelerated observer under consideration in this paper is described in Sec. III. The nonlocal electromagnetic measurements of the observer are studied in Secs. IV and V. Section VI contains a brief discussion of our results.

\section{NONLOCALITY}

According to the hypothesis of locality, the electromagnetic radiation field measured by an accelerated observer is given by

$$
F_{(\alpha)(\beta)}(\tau)=F_{\mu \nu}(t(\tau), \mathbf{x}(\tau)) \lambda_{(\alpha)}^{\mu}(\tau) \lambda_{(\beta)}^{\nu}(\tau),
$$

which is the projection of the Faraday tensor onto the tetrad of the accelerated observer. To go beyond the hypothesis of locality, one must find a more general relationship between the measurements of the accelerated observer $\mathcal{F}_{(\alpha)(\beta)}(\tau)$ and the infinite class of momentarily comoving inertial observers $F_{(\alpha)(\beta)}(\tau)$. The most general linear relationship between $\mathcal{F}_{(\alpha)(\beta)}(\tau)$ and $F_{(\alpha)(\beta)}(\tau)$ consistent with causality is [15]

$$
\mathcal{F}_{(\alpha)(\beta)}(\tau)=F_{(\alpha)(\beta)}(\tau)+\int_{\tau_{0}}^{\tau} K_{(\alpha)(\beta)}(\gamma)(\delta)\left(\tau, \tau^{\prime}\right) F_{(\gamma)(\delta)}\left(\tau^{\prime}\right) d \tau^{\prime},
$$

where $\tau_{0}$ is the instant at which the acceleration is turned on and $K_{(\alpha)(\beta)}(\gamma)(\delta)$ is a kernel that is expected to be proportional to the acceleration of the observer. For a radiation field with $\chi / \mathcal{L} \rightarrow 0$, the nonlocal part of the ansatz (14) is expected to vanish. The nonlocal ansatz (14) deals only with spacetime scalars and is thus manifestly invariant under inhomogeneous Lorentz transformations of the background spacetime.

The nonlocal part in Eq. (14) has the form of an average over the past worldline of the accelerated observer; in the JWKB limit, the nonlocal part disappears and we recover the hypothesis of locality (13). Equation (14) expresses a Volterra integral equation of the second kind. According to Volterra's theorem, the relationship between $\mathcal{F}_{(\alpha)(\beta)}$ and $F_{(\alpha)(\beta)}$ is unique in the space of continuous functions [24]. Volterra's theorem has been extended to the Hilbert space of squareintegrable functions by Tricomi [25]. It is useful to rewrite Eqs. (13) and (14) in matrix form by replacing the Faraday tensors by six-vectors consisting of electric and magnetic fields. Thus Eq. (13) may be reexpressed as $\hat{F}=\Lambda F$, where
$F_{(\alpha)(\beta)} \rightarrow \hat{F}, F_{\mu \nu} \rightarrow F$, and $\Lambda$ is a $6 \times 6$ matrix constructed from the local tetrad frame. It follows that Eq. (14) can be written as

$$
\hat{\mathcal{F}}(\tau)=\hat{F}(\tau)+\int_{\tau_{0}}^{\tau} \hat{K}\left(\tau, \tau^{\prime}\right) \hat{F}\left(\tau^{\prime}\right) d \tau^{\prime}
$$

where $\hat{K}$ is a $6 \times 6$ matrix.

To find the kernel in Eq. (15), we assume that no accelerated observer can ever be comoving with an electromagnetic radiation field. This extends to all observers an important consequence of Lorentz invariance: a basic radiation field can never stand completely still with respect to any inertial observer. That is, if the incident radiation is nonconstant (as it must be) for inertial observers, it will be nonconstant for any accelerated observer. Equivalently, if an accelerated observer measures a constant electromagnetic radiation field, i.e., $\hat{\mathcal{F}}(\tau)=\hat{F}\left(\tau_{0}\right)$ in Eq. (15), then the inertial observers must also measure constant fields, i.e., $F$ must be constant as well. The Volterra-Tricomi uniqueness theorem then ensures that our physical requirement is satisfied for any radiation field: a variable field will never be constant for any observer. Inserting these conditions in Eq. (15), we find

$$
\Lambda\left(\tau_{0}\right)=\Lambda(\tau)+\int_{\tau_{0}}^{\tau} \hat{K}\left(\tau, \tau^{\prime}\right) \Lambda\left(\tau^{\prime}\right) d \tau^{\prime}
$$

which may be used to determine the kernel on the basis of our physical postulate. However, Eq. (16) is not sufficient to determine the kernel uniquely. A detailed examination of the possible kernels $[16,17]$ has revealed that the only acceptable solution is the kinetic kernel given by $\hat{K}\left(\tau, \tau^{\prime}\right)=k\left(\tau^{\prime}\right)$. In this case, Eq. (16) immediately implies that

$$
k(\eta)=-\frac{d \Lambda(\eta)}{d \eta} \Lambda^{-1}(\eta)
$$

This kernel is directly proportional to the observer's acceleration and vanishes when it is turned off. Moreover, it is constant for the case of uniform acceleration. A nonlocal theory of accelerated observers has been developed on the basis of this unique kernel [15-18] and nonlocal Maxwell's equations have been discussed in [19].

It is important to recognize that the hypothesis of locality is nevertheless an integral part of the nonlocal theory described here: in the eikonal limit $\chi / \mathcal{L} \rightarrow 0$, the nonlocal theory reduces to the standard theory based on the hypothesis of locality. This is analogous to the correspondence between wave mechanics and classical mechanics. The idea of such a correspondence will be employed throughout this paper; for instance, we will assume that accelerated observers can perform spatial and temporal measurements that are essentially consistent with the hypothesis of locality (see Sec. III). A nonlocal treatment will be required only if the wave phenomena involved are such that $x / \mathcal{L}$ is not negligibly small and hence cannot be ignored. 


\section{LINEARLY ACCELERATED SYSTEMS}

In this paper we are interested in the electromagnetic measurements of a linearly accelerated observer. For the sake of concreteness, we assume that the observer moves uniformly with velocity $\mathbf{v}=v_{0} \hat{\mathbf{z}}$ in the background global inertial frame with coordinates $x^{\alpha}=(t, x, y, z)$ according to $x=x_{0}, y$ $=y_{0}$ and $z=z_{0}+v_{0} t$ for $-\infty<t<0$ and at $t=0$ is forced to accelerate with acceleration $g(\tau)>0$ along the positive $z$ direction. Henceforth we use units such that $c=1$, unless specified otherwise. The observer carries a natural orthonormal tetrad frame $\lambda_{(\alpha)}^{\mu}$ given by

$$
\begin{array}{ll}
\lambda_{(0)}^{\mu} & =(C, 0,0, S), \quad \lambda_{(1)}^{\mu}=(0,1,0,0), \\
\lambda_{(2)}^{\mu} & =(0,0,1,0), \quad \lambda_{(3)}^{\mu}=(S, 0,0, C),
\end{array}
$$

where $C=\cosh \theta, S=\sinh \theta$, and

$$
\theta=\theta_{0}+u(\tau) \int_{0}^{\tau} g\left(\tau^{\prime}\right) d \tau^{\prime} .
$$

Here $\tanh \theta_{0}=v_{0}$ and $u(\tau)$ is the unit step function such that $u(\tau)=1$ for $\tau>0$ and $u(\tau)=0$ for $\tau<0$. The orthonormal frame of the observer is nonrotating, i.e., it is Fermi-Walker transported along the worldline of the observer. We recall that a Fermi-Walker transported four-vector $v^{\mu}$ carried along a worldline $x^{\mu}(\tau)$ obeys the transport equation

$$
\frac{d v^{\mu}}{d \tau}=\left(u^{\mu} a^{\nu}-u^{\nu} a^{\mu}\right) v_{\nu},
$$

where $u^{\mu}=\lambda_{(0)}^{\mu}$ is the four-velocity and $a^{\mu}=d u^{\mu} / d \tau$ is the four-acceleration vector of the worldline. Thus each leg of the tetrad (18) satisfies Eq. (20); moreover, we note that $a_{\mu} \lambda_{(3)}^{\mu}=u(\tau) g(\tau)$, where we have taken the signature of the Minkowski metric to be +2 . The magnitude of the fouracceleration vector $g$ is related to the magnitude of the threeacceleration vector $A$ by $g=A \gamma^{3}$, where $\gamma$ is the Lorentz factor.

We assume for the sake of simplicity that the acceleration of the observer $g(\tau)$ is uniform and equal to $g_{0}$ for $0<\tau$ $<\tau_{f}$, but vanishes otherwise. That is

$$
g(\tau)=g_{0}\left[u(\tau)-u\left(\tau-\tau_{f}\right)\right] .
$$

Therefore, the observer starts from its position $\left(x_{0}, y_{0}, z_{0}\right)$ at $t=0$ and accelerates uniformly according to

$$
\begin{gathered}
t=\frac{1}{g_{0}}\left[\sinh \left(\theta_{0}+g_{0} \tau\right)-\sinh \theta_{0}\right], \quad x=x_{0}, \quad y=y_{0}, \\
z=z_{0}+\frac{1}{g_{0}}\left[\cosh \left(\theta_{0}+g_{0} \tau\right)-\cosh \theta_{0}\right]
\end{gathered}
$$

until the time $t_{f}$, where $g_{0} t_{f}=\sinh \left(\theta_{0}+g_{0} \tau_{f}\right)-\sinh \theta_{0}$. For $t$ $>t_{f}$, the observer moves uniformly with speed $\beta_{f}=\tanh \left(\theta_{0}\right.$ $\left.+g_{0} \tau_{f}\right)$,

$$
x=x_{0}, \quad y=y_{0}, \quad z=z_{f}+\beta_{f}\left(t-t_{f}\right),
$$

where $z_{f}$ is given by $g_{0}\left(z_{f}-z_{0}\right)=\cosh \left(\theta_{0}+g_{0} \tau_{f}\right)-\cosh \theta_{0}$ using Eq. (22). The proper time of the observer for $t>t_{f}$ is given by

$$
\tau=\tau_{f}+\frac{t-t_{f}}{\cosh \left(\theta_{0}+g_{0} \tau_{f}\right)} .
$$

It follows from the results of the previous section that in this case

$$
\Lambda=\left[\begin{array}{cc}
U & V \\
-V & U
\end{array}\right], \quad U=\left[\begin{array}{ccc}
C & 0 & 0 \\
0 & C & 0 \\
0 & 0 & 1
\end{array}\right], \quad V=S I_{3},
$$

where $I_{i},\left(I_{i}\right)_{j k}=-\epsilon_{i j k}$, is a $3 \times 3$ matrix proportional to the operator of infinitesimal rotations about the $x^{i}$ axis. According to the postulates of the nonlocal theory of accelerated observers, the fields as measured by the linearly accelerated observer are given by Eq. (14), where the kernel, given by Eq. (17), reduces in this case to

$$
k(\tau)=-g(\tau)\left[\begin{array}{cc}
0 & I_{3} \\
-I_{3} & 0
\end{array}\right] .
$$

In the standard theory of relativity, the field as measured by the accelerated observer is determined via the hypothesis of locality and for the specific case of the linearly accelerated observer described in this section

$$
\begin{gathered}
E_{(1)}=C E_{1}-S B_{2}, \quad B_{(1)}=C B_{1}+S E_{2}, \\
E_{(2)}=C E_{2}+S B_{1}, \quad B_{(2)}=C B_{2}-S E_{1}, \\
E_{(3)}=E_{3}, \quad B_{(3)}=B_{3},
\end{gathered}
$$

where $(C, S)=\left(\cosh \theta_{0}, \sinh \theta_{0}\right)$ for $t<0,(C, S)=\left(\cosh \left(\theta_{0}\right.\right.$ $\left.\left.+g_{0} \tau\right), \sinh \left(\theta_{0}+g_{0} \tau\right)\right)$ for $0 \leqslant t \leqslant t_{f}$ and $(C, S)=\left(\cosh \left(\theta_{0}\right.\right.$ $\left.\left.+g_{0} \tau_{f}\right), \sinh \left(\theta_{0}+g_{0} \tau_{f}\right)\right)$ for $t>t_{f}$. However, according to the nonlocal theory the electric field is given by

$$
\begin{gathered}
\mathcal{E}_{(1)}=E_{(1)}+u(\tau) \int_{0}^{\tau} g\left(\tau^{\prime}\right) B_{(2)}\left(\tau^{\prime}\right) d \tau^{\prime}, \\
\mathcal{E}_{(2)}=E_{(2)}-u(\tau) \int_{0}^{\tau} g\left(\tau^{\prime}\right) B_{(1)}\left(\tau^{\prime}\right) d \tau^{\prime}, \\
\mathcal{E}_{(3)}=E_{(3)} .
\end{gathered}
$$

Similarly, the nonlocal magnetic field is given by

$$
\begin{aligned}
& \mathcal{B}_{(1)}=B_{(1)}-u(\tau) \int_{0}^{\tau} g\left(\tau^{\prime}\right) E_{(2)}\left(\tau^{\prime}\right) d \tau^{\prime}, \\
& \mathcal{B}_{(2)}=B_{(2)}+u(\tau) \int_{0}^{\tau} g\left(\tau^{\prime}\right) E_{(1)}\left(\tau^{\prime}\right) d \tau^{\prime},
\end{aligned}
$$




$$
\mathcal{B}_{(3)}=B_{(3)} .
$$

It follows that the components of the electric and magnetic fields parallel to the direction of motion of the observer remain the same. Moreover, for $\tau>\tau_{f}$ the observer moves uniformly yet its measurement of the electromagnetic field yields in addition to the standard result a new constant component that is in effect the memory of the past acceleration of the observer.

The measurements of the accelerated observer for the case of a perpendicularly incident plane-polarized Gaussian pulse of electromagnetic radiation were numerically investigated in [26] on the basis of the hypothesis of locality, namely, Eqs. (27)-(29). The same situation has recently been studied in connection with the nonlocal measurements of the observer involving Eqs. (30)-(35); in fact, an extension of the previous work [26] and a detailed numerical analysis of nonlocality in this case are contained in [27].

To illustrate further the physical consequences of the nonlocal theory, two rather distinct situations will be explicitly worked out in the rest of this paper. In the first case ("parallel incidence"), discussed in the next section, the electromagnetic radiation propagates along the $z$ axis. This situation is analogous to the acceleration of grains by a high-intensity femtosecond laser pulse; indeed, the results of this work should be compared and contrasted with the analysis of the experimental situation presented in $[22,23]$. The second case ("perpendicular incidence"), discussed in Sec. V, involves a pulse of plane-polarized radiation that propagates along the $x$ axis; the nonlocal measurements of the accelerated observer are compared with the standard theory. To simplify the analysis, we take advantage of the fact that all of the field operations considered in this work are linear. Therefore, it suffices to focus attention on a generic Fourier component of the incident pulse. Moreover, we use complex fields whose real parts correspond to the measured fields. Thus to recover the actual predictions of the theory, one must take the real part of the Fourier sum of the results given in Secs. IV and V.

\section{PARALLEL INCIDENCE}

Imagine a plane monochromatic wave of frequency $\omega$ given in the circular polarization basis by

$$
\mathbf{E}=E_{0} \mathbf{e}_{ \pm} e^{i \omega(z-t)}, \quad \mathbf{B}=E_{0} \mathbf{b}_{ \pm} e^{i \omega(z-t)},
$$

where $\mathbf{e}_{ \pm}=(\hat{\mathbf{x}} \pm i \hat{\mathbf{y}}) / \sqrt{2}, \mathbf{b}_{ \pm}=\mp i \mathbf{e}_{ \pm}$, and $E_{0}(\omega)$ is the amplitude of the wave. The wave propagates along the $z$ direction and at $t=0$ impinges on a grain ("observer") at $z=z_{0}$ that accelerates along the $z$ axis with initial velocity $v_{0}$. For $0 \leqslant \tau$ $\leqslant \tau_{f}$, it follows from Eq. (22) that in the six-vector notation of Sec. II

$$
F(\tau)=E_{0}(\omega) \phi(\theta)\left[\begin{array}{l}
\mathbf{e}_{ \pm} \\
\mathbf{b}_{ \pm}
\end{array}\right]
$$

where $\phi$ is given by

$$
\phi(\theta)=e^{i \omega z_{0}} \exp \left[i \frac{\omega}{g_{0}}\left(e^{-\theta}-e^{-\theta_{0}}\right)\right]
$$

and $\theta=\theta_{0}+g_{0} \tau$. Moreover, $\hat{F}=\Lambda F$ implies that in this case

$$
\hat{F}=e^{-\theta} F .
$$

This result together with the kernel (26) can be substituted in Eq. (15) with $\tau_{0}=0$ and after a simple integration the nonlocal result is

$$
\hat{\mathcal{F}}(\tau)=E_{0} \Phi(\tau)\left[\begin{array}{l}
\mathbf{e}_{ \pm} \\
\mathbf{b}_{ \pm}
\end{array}\right]
$$

where

$$
\Phi(\tau)=e^{-\theta(\tau)} \phi(\theta)+i \frac{g_{0}}{\omega}\left[\phi(\theta)-\phi\left(\theta_{0}\right)\right] .
$$

It is important to note that the nonlocal theory, just as in the local case, does not introduce any coupling between the photon helicity and the acceleration of the observer. The nonlocal contribution to $\Phi$ is given by $i\left(g_{0} / \omega\right)\left[\phi(\theta)-\phi\left(\theta_{0}\right)\right]$ in Eq. (41); as expected, it is proportional to $\chi / \mathcal{L}_{0}=g_{0} / \omega$, which for the experiments described in $[22,23]$ is negligibly small and of the order of $10^{-5}$.

In connection with the treatment of [22,23], it is interesting to note that in Eq. (22), one can expand in powers of $g_{0} \tau \ll c$ to get

$t=\gamma_{0} \tau\left(1+\frac{1}{2} \beta_{0} \frac{g_{0} \tau}{c}+\cdots\right), \quad z=z_{0}+\gamma_{0} \tau\left(v_{0}+\frac{1}{2} g_{0} \tau+\cdots\right)$,

where $\beta_{0}=v_{0} / c, \gamma_{0}$ is the corresponding Lorentz factor and in Eq. (38)

$$
e^{-\theta_{0}}=\sqrt{\frac{1-\beta_{0}}{1+\beta_{0}}} .
$$

It follows that

$$
z=z_{0}+v_{0} t+\frac{1}{2} A_{0} t^{2}+\cdots,
$$

where $A_{0}=g_{0} / \gamma_{0}^{3}$ is the magnitude of the initial threedimensional acceleration vector. Using these results, $\phi$ and $\Phi$ in Eq. (41) can be expressed as

$$
\begin{aligned}
\phi= & e^{i(\omega / c)\left(-c t+z_{0}+v_{0} t+A_{0} t^{2} / 2+\cdots\right)}, \\
\Phi= & {\left[\sqrt{\frac{1-\beta_{0}}{1+\beta_{0}}}+i \frac{g_{0}}{c \omega}+\frac{g_{0}}{c^{2}}\left(-c t+v_{0} t+\frac{1}{2} A_{0} t^{2}+\cdots\right)\right] \phi } \\
& -i \frac{g_{0}}{c \omega} e^{i(\omega / c) z_{0}},
\end{aligned}
$$

in general agreement with [23]. One can thus draw the conclusion that the nonlocal contribution to the field that sets the charged particles in the grain in motion has an amplitude of order $10^{-5}$ compared to the standard local theory for the experiments reported in $[22,23]$. The reflected wave is therefore expected to be affected at essentially the same negligibly small level of $\sim 10^{-5}$. It is this reflected wave that is detected and analyzed in practice $[22,23]$. The nonlocal theory in this case would thus appear to be trivially consistent with the results of experiments reported in $[22,23]$, since the nonlo- 
cality enters the analysis at the insignificant level of $g_{0} / \omega$ $\sim 10^{-5}$.

Nonlocal effects may not be negligible in future experiments using macrophysical accelerated systems [22,23]. A remark is therefore in order here regarding the relevance of our present calculations that are based on a single plane wave. It should be emphasized that further extensive calculations using wave packets would be necessary in order that the nonlocal theory could be properly compared with experimental data regarding accelerated plasmas generated by an incident femtosecond laser pulse $[22,23]$. What is measured in such experiments is the shape of the reflected pulse $[22,23]$; therefore, to determine the anticipated contribution of nonlocality, one should consider an incident pulse in the accelerated frame that is a Fourier sum of terms of the form (41). One must then take into account the interaction of this incident radiation with the accelerated medium via an effective reflectivity function as in [22]. The theoretical determination of the reflected pulse in the laboratory frame would then require an inverse nonlocal transformation-given in its general form by Eq. (19) of [19]—and hence further detailed considerations that are beyond the scope of the present paper.

For $\tau \geqslant \tau_{f}$, the measurements of the observer are given by Eq. (40), where $\Phi(\tau)$ for $\tau \geqslant \tau_{f}$ can be expressed as

$$
\Phi(\tau)=e^{-\theta_{f}} \widetilde{\phi}(\tau)+i \frac{g_{0}}{\omega}\left[\phi\left(\theta_{f}\right)-\phi\left(\theta_{0}\right)\right]
$$

Here $\theta_{f}=\theta_{0}+g_{0} \tau_{f}$ and $\widetilde{\phi}(\tau)$ is given by

$$
\widetilde{\phi}(\tau)=\exp \left\{i \omega\left[z_{f}-t_{f}-\sqrt{\frac{1-\beta_{f}}{1+\beta_{f}}}\left(\tau-\tau_{f}\right)\right]\right\} .
$$

The second term in $\Phi(\tau)$ is the constant memory of the observer's past acceleration.

\section{PERPENDICULAR INCIDENCE}

Consider a linearly polarized plane monochromatic wave of frequency $\omega$ propagating in the $x$ direction. The electric and magnetic fields are given by

$$
\mathbf{E}=E_{0} e^{i \omega(x-t)} \hat{\mathbf{z}}, \quad \mathbf{B}=-E_{0} e^{i \omega(x-t)} \hat{\mathbf{y}} .
$$

At $t=0$ the wave impinges upon a grain ("observer") at $\left(x_{0}, y_{0}, z_{0}\right)$ that has been prearranged to accelerate along the $z$ direction with initial velocity $v_{0}$. To determine the field measured by the observer, it is useful to assume that $0 \leqslant \tau \leqslant \tau_{f}$ and define $\chi(\theta)$,

$$
\chi(\theta)=E_{0} e^{i \omega x_{0}} \exp \left[-i \frac{\omega}{g_{0}}\left(\sinh \theta-\sinh \theta_{0}\right)\right],
$$

where $\theta=\theta_{0}+g_{0} \tau$. The measured components of the electromagnetic field according to the nonlocal theory are then

$$
\begin{array}{r}
\mathcal{E}_{(1)}=\sinh \theta \chi(\theta)-\int_{\theta_{0}}^{\theta} \cosh \theta^{\prime} \chi\left(\theta^{\prime}\right) d \theta^{\prime}, \\
\mathcal{E}_{(2)}=0, \mathcal{E}_{(3)}=\chi(\theta), \mathcal{B}_{(1)}=\mathcal{B}_{(3)}=0, \text { and }
\end{array}
$$

$$
\mathcal{B}_{(2)}=-\cosh \theta \chi(\theta)+\int_{\theta_{0}}^{\theta} \sinh \theta^{\prime} \chi\left(\theta^{\prime}\right) d \theta^{\prime}
$$

It is simple to work out the integral in Eq. (50) and the result is

$$
\mathcal{E}_{(1)}=\sinh \theta \chi(\theta)-i \frac{g_{0}}{\omega}\left[\chi(\theta)-\chi\left(\theta_{0}\right)\right],
$$

where $\chi\left(\theta_{0}\right)=E_{0} \exp \left(i \omega x_{0}\right)$. The integral in Eq. (51) can be evaluated by expanding the exponential function in $\chi\left(\theta^{\prime}\right)$ in powers of $\sinh \theta^{\prime}$ and then using formulas 2.412 on p. 93 of [28].

For $\tau \geqslant \tau_{f}$, the fields measured by the uniformly moving observer are

$$
\mathcal{E}_{(1)}=\sinh \theta_{f} \tilde{\chi}(\tau)-i \frac{g_{0}}{\omega}\left[\chi\left(\theta_{f}\right)-\chi\left(\theta_{0}\right)\right]
$$

$\mathcal{E}_{(2)}=0, \mathcal{E}_{(3)}=\tilde{\chi}, \mathcal{B}_{(1)}=\mathcal{B}_{(3)}=0$, and

$$
\mathcal{B}_{(2)}=-\cosh \theta_{f} \tilde{\chi}(\tau)+\int_{\theta_{0}}^{\theta_{f}} \sinh \theta^{\prime} \chi\left(\theta^{\prime}\right) d \theta^{\prime} .
$$

Here $\tilde{\chi}(\tau)$ is given by

$$
\tilde{\chi}(\tau)=E_{0} e^{i \omega\left[x_{0}-t_{f}-\gamma_{f}\left(\tau-\tau_{f}\right)\right]},
$$

where $\gamma_{f}=\cosh \theta_{f}$. The constant terms in Eq. (53) and (54) are the remnants of the observer's accelerated history.

\section{DISCUSSION}

We have examined some of the observational consequences of the nonlocal theory of accelerated observers for the case of linearly accelerated systems. As in the standard local theory, the electromagnetic radiation field parallel to the direction of motion remains unchanged and, moreover, there is no coupling between the helicity of the radiation and the translational acceleration of the observer [29,30]. The observer's nonlocal determination of the electromagnetic radiation field has been compared and contrasted with the standard local ansatz. The results are consistent with a relative nonlocal contribution to the field given essentially by the amplitude $\chi / \mathcal{L}$, which turns out to be negligibly small for the experiments reported in [22,23]. Moreover, for an observer that has resumed uniform motion, the constant electromagnetic memory of past acceleration has been studied. The observation of such nonlocal effects may become possible with methods that use high-power laser systems to generate large accelerations [20-23].

\section{ACKNOWLEDGMENTS}

Thanks are due to C. Chicone and J. Hauck for helpful discussions. I am grateful to G. Schäfer and R. Sauerbrey for their constructive comments. 
[1] J. D. Jackson, Classical Electrodynamics, 2nd ed. (Wiley, New York, 1975).

[2] A. Einstein, The Meaning of Relativity (Princeton University Press, Princeton, NJ, 1955).

[3] B. Mashhoon, Phys. Lett. A 143, 176 (1990).

[4] B. Mashhoon, Phys. Lett. A 145, 147 (1990).

[5] B. Mashhoon, in Relativity in Rotating Frames, edited by G. Rizzi and M. L. Ruggiero (Kluwer Academic Publishers, Dordrecht, 2003), pp. 43-55.

[6] J. C. Hauck and B. Mashhoon, Ann. Phys. (Leipzig) 12, 275 (2003).

[7] M. S. Gupta, Am. J. Phys. 43, 1087 (1975).

[8] W. Moreau, Am. J. Phys. 60, 561 (1992).

[9] R. Neutze and W. Moreau, Phys. Lett. A 179, 389 (1993).

[10] B. Rothenstein and A. De Sabata, Eur. J. Phys. 19, 569 (1998).

[11] B. Mashhoon, Phys. Lett. A 122, 299 (1987).

[12] R. Neutze and W. Moreau, Phys. Lett. A 183, 141 (1993).

[13] N. Bohr and L. Rosenfeld, K. Dan. Vidensk. Selsk. Mat. Fys. Medd. 12, No. 8, (1933); translated in Quantum Theory and Measurement, edited by J. A. Wheeler and W. H. Zurek (Princeton University Press, Princeton, NJ, 1983).

[14] N. Bohr and L. Rosenfeld, Phys. Rev. 78, 794 (1950).

[15] B. Mashhoon, Phys. Rev. A 47, 4498 (1993).
[16] C. Chicone and B. Mashhoon, Ann. Phys. (Leipzig) 11, 309 (2002).

[17] C. Chicone and B. Mashhoon, Phys. Lett. A 298, 229 (2002).

[18] F. W. Hehl and Y. N. Obukhov, Foundations of Classical Electrodynamics (Birkhäuser, Boston, 2003).

[19] B. Mashhoon, Ann. Phys. (Leipzig) 12, 586 (2003).

[20] G. A. Mourou, C. P. J. Barty, and M. D. Perry, Phys. Today 51(1), 22 (1998).

[21] D. Umstadter, Phys. Plasmas 8, 1774 (2001).

[22] R. Sauerbrey, Phys. Plasmas 3, 4712 (1996).

[23] G. Schäfer and R. Sauerbrey, e-print astro-ph/9805106.

[24] V. Volterra, Theory of Functionals and of Integral and IntegroDifferential Equations (Dover, New York, 1959).

[25] F. G. Tricomi, Integral Equations (Interscience, New York, 1957).

[26] B. Mashhoon, Phys. Lett. A 122, 67 (1987).

[27] J. C. Hauck, Ph.D. thesis, University of Missouri-Columbia, 2003.

[28] I. S. Gradshteyn and I. M. Ryzhik, Table of Integrals, Series and Products (Academic Press, New York, 1980).

[29] B. Mashhoon, Class. Quantum Grav. 17, 2399 (2000).

[30] D. Bini, C. Cherubini, and B. Mashhoon, Class. Quantum Grav. 21, 3893 (2004). 